\title{
A simple statistical procedure for the analysis of radon anomalies associated with seismic activity
}

\author{
Hugo Gonçalves Silva ${ }^{1,{ }^{\star}}$, Mourad Bezzeghoud ${ }^{1}$, Maria Manuela Oliveira ${ }^{2}$, \\ António Heitor Reis ${ }^{1}$, Rui Namorado Rosa ${ }^{1}$
}

\author{
${ }^{1}$ University of Évora, School of Sciences and Technology (ECT), Geophysics Centre of Évora and Physics Department, Évora, Portugal \\ ${ }^{2}$ University of Évora, School of Sciences and Technology (ECT), Mathematics Department, Évora, Portugal
}

\author{
Article history \\ Received February 8, 2012; accepted November 8, 2012. \\ Subject classification: \\ Atmosphere: Composition and Structure, Geochemical exploration, Radon emissions, Seismic precursors, Seismic activity, Seismic risk.
}

\section{ABSTRACT}

This study presents an analysis of data from various radon anomalies that were compiled by Toutain and Baubron [1999], to investigate their relationships with the earthquake parameters of magnitude and distance from epicenter. The simple methodology applied here reveals significant and positive correlation between the duration of the radon anomalies and the ratio between the earthquake preparation radius and the distance between the sensor and the event epicenter. This shows an important relationship between seismic activity and duration of radon anomalies at a local scale. The consequences and implications of this relationship are discussed.

\section{Introduction}

The study of earthquake precursors has a long history that goes back to the late 19th century [e.g., Kagan and Knopoff 1987, Geller 1991, Wyss 1991; and references therein]. In the last few years, the study of these phenomena has been gaining new widespread interest. The reason for this is clearly due to the valuable tools that are being developed in this field to complement existing hazard assessment facilities. In the literature, it is possible to find a number of different effects with many occurrences [e.g., Biagi et al. 2006, Ouzounov et al. 2007, Chauhan et al. 2009, Silva et al. 2011]. The interested reader is referred to a recent review by Cicerone et al. [2009].

Many studies have attributed these effects to radon emanations that can be caused by variations in the bedrock strain and the soil permeability during the preparatory phase of earthquakes, and particularly by severe changes near to the end of this phase [see, e.g., Pulinets and Ouzounov 2011]. For example, according to Harrison et al. [2010], the radon released in this process can ionize the lower atmosphere, to produce a considerable reduction in the atmospheric electrical field.

Due to its relevance, comprehensive analyses of radon emanations are crucial, and many studies have dealt with this issue. In particular, Toutain and Baubron [1999] carried out a very extensive review of various degassing processes in relation to seismic activity for different gases (especially radon), with specific attention to the multiple factors that can influence these processes (mainly weather conditions). As a discussion of these aspects is not within the scope of the present study, the reader is referred to Toutain and Baubron [1999], where many further details can be found.

In the present study, we applied a simple statistical procedure that was recently developed by Silva et al. [2012] to the radon anomalies associated with seismic activity that were compiled by Toutain and Baubron [1999]. We focused, in particular, on a study of the relationship between the duration of the anomalies and the magnitude of the earthquake and distance from its epicenter. These anomalies are defined based on deviations in the radon levels from the soil or groundwater. Here, after presenting the database used and describing our methodology in Section 2, we apply this to the occurrences reported by Toutain and Baubron [1999], including together both the soil and groundwater radon anomalies, as they are linked by the same physical processes discussed in Section 3. Our analysis indicates that the duration of the radon anomalies is linearly related to the earthquake preparation radius (which is exponentially dependent on its magnitude), and is inversely related to the distance from the earthquake epicenter to the radon-measuring site.

\section{Data}

In this study, we have analyzed the various radon anomalies that were compiled by Toutain and Baubron [1999]. Their review includes nearly 150 anomalies of different geochemical indicators, including: $\mathrm{Rn}, \mathrm{H}_{2}, \mathrm{~N}_{2}$, Ar, $\mathrm{He}, \mathrm{CH}_{4}, \mathrm{H}_{2} \mathrm{~S}$ and $\mathrm{Hg}$ (gas). Nearly $72 \%$ of these anomalies are related to radon, which provided a significant number of cases to which we can apply our methodology. These are defined as size- 
able deviations from the mean background levels of radon, $\bar{x}$, which is a typically criterion used in various studies that considers a given measurement as an anomaly when the radon levels rise above $\bar{x}+2 \sigma$, with $\sigma$ as the standard deviation. This is a well-established criterion in the literature that provides good support for our analysis, although, unfortunately, the values of $\bar{x}$ and $\sigma$ are not presented in the review by Toutain and Baubron [1999], because normally they are not mentioned in the original papers.

Most of the anomalies (83\%) correspond to groundwater measurements, with $10 \%$ from soil measurements, and $7 \%$ measured using both groundwater and soil observations. These values should imply a dominance of the groundwater processes in our results; however, interestingly, Virk and Baljinder [1994] showed that groundwater and soil radon measurements show similar behaviors as earthquake precursors. Thus our dataset should be representative of the general radon emanation dynamics in connection with earthquake occurrences.

The measurements were made for intermediate to longterm periods (more than 1 year of observations), and different techniques were used. The soil radon anomalies were measured using the track-etch method [Virk and Baljinder 1994], which is usually a low-frequency technique (integrated over one or two weeks) that does not allow the correct estimation of the anomaly duration and its starting time. On the other hand, groundwater measurements typically consider daily sampling from natural springs, and a subsequent closedcircuit system enables the collected gases from these samples to pass through a detector; e.g., a $\mathrm{ZnS}(\mathrm{Ag})$ cell. This detector measures the number of decays recorded for a given period after radon and its progeny reach equilibrium [Virk and Baljinder 1994]. This technique was used for $90 \%$ of our dataset, and it allows better control of the anomaly duration and starting time.

It is important to note that $63 \%$ of the anomalies considered in the present study corresponded to $M \geq 5$ earthquakes. Indeed, different precursor behaviors would be expected for small and large earthquakes, and a careful analysis regarding this will be carried out in the future.

For each radon anomaly, Toutain and Baubron [1999] presented (for the information that is available): the magnitude, $M$, and depth of the earthquake, $z(\mathrm{~km})$; the distance from the earthquake epicenter to the measurement site, $D$ $(\mathrm{km})$; the relative amplitude of the radon measurement, $\delta a$ $(\%)$; the duration of the anomaly, $d$ (days); and the time interval between the beginning of the anomaly and the occurrence of the earthquake, $\delta t$ (days). We selected 109 anomalies where that the earthquake parameters $M$ and $D$ are available (we did not considered $z$ in the analysis, and thus this parameter was ignored), along with at least one of the three parameters of the radon anomalies, $\delta a, d$, and $\delta t$. The ranges of theses parameters are: $M=[1.9,8.1] ; D=[1,1000](\mathrm{km})$; $\delta a=[-80,1200](\%) ; d=[1,1370]($ days $) ; \delta t=[1,180]$ (days).

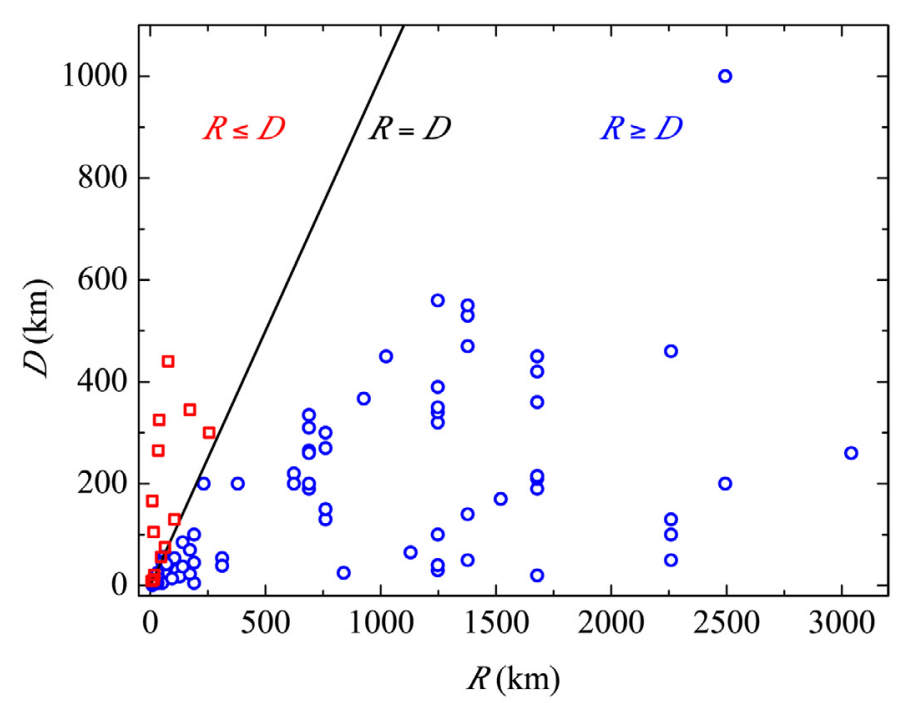

Figure 1. Representation of all radon data points from Toutain and Baubron [1999] in the $R-D$ plane, where $R$ is the earthquake preparation radius, and $D$ the distance $(\mathrm{km})$ from the epicenter to the measuring site.

\section{Methodology}

We will now focus on a description of the methodology applied. First, we should note that when analyzing the possible effects of an earthquake on anomalous radon emanations, two parameters must be considered together: the event magnitude, $M$, and the distance from the epicenter to the measuring site, $D$. This can be clarified when two earthquakes of the same magnitude are considered, where they occur at different distances from a measuring site: it is expected that the nearer event will influence the radon emanations more significantly than the farther event, such that we cannot compare them directly. This is because, in principle, both of the earthquakes would be expected to generate similar deformation fields, while the deformation caused by the nearer one to the measurement site is expected to be stronger, thereby causing a substantial increase in the local soil permeability, and enabling greater radon emanation, as compared to the farther event. Thus, it is important to develop a parameter that is suitable to simultaneously account for both the effects of $M$ and $D$, as these two parameters by themselves are insufficient to describe the significance of an earthquake with respect to the radon levels at a measuring site.

On this basis, we considered the concept of the earthquake preparation radius, $R$, that depends on $M$ [see Dobrovolsky et al. 1979]. It is assumed that there should be an approximately circular region around the epicenter of an earthquake that undergoes elastic crustal deformation prior to seismic events, with this radius estimated as $R \approx 10^{0.43 M}$ $(\mathrm{km})$. Bearing in mind these observations, we can assume that for relevant events, the radon measurement site will fall within a circle of radius $R$ that is centered at the seismic epicenter, such that $R \geq D$. Indeed, we have represented the 109 occurrences of radon anomalies that are relevant to the present study in the $R-D$ plane in Figure 1. From Figure 1, it is clear that more than $84 \%$ of the events obey the $R \geq D$ con- 
dition. In the remaining $16 \%, 10$ anomalies almost coincide as $R=D$ (black line), while seven of the anomalies correspond to microseismicity data from the same study, Virk [1995], as cited by Toutain and Baubron [1999].

In line with the above arguments, Silva et al. [2012] introduced a dimensionless parameter, $S$, that was defined as follows:

$$
S=\frac{R}{D}-1,
$$

where $S$ should be positive, or very small if negative, for occurrences considered to be relevant to the present study; i.e., those that obey the $R \geq D$ condition. In the present analysis, we used the restrictive criterion of $S \geq 0$ for the relevant cases. Figure 2 shows the parameters that characterized the observed radon anomalies; namely, $\delta a$ (Figure 2a), $d$ (Figure $2 \mathrm{~b}$ ), and $\delta t$ (Figure 2c), as a function of $S$ for these cases. In the $S$-d plot (Figure $2 \mathrm{~b}$ ), it is possible to identify a clear outlier point for $S \cong 16.38$ and $d=1370$ days, which corresponds to an earthquake with $M=7.8$ at $130 \mathrm{~km}$ from the sensor, and a radon anomaly with $\delta a=-40 \%$ and no information about $\delta t$, as reported by Hauksson [1981]. Indeed, Toutain and Baubron [1999] noted that in areas with frequent highmagnitude earthquakes, more than one event might cause an apparently single anomaly. In the particular case of this outlier, where $\delta t$ was not identified, it might have been related to either the Haicheng (February 4, 1975, M 7.3) or the Tangshan (July 28, 1976, M 7.8) earthquake [Toutain and Baubron 1999], and for that reason this point was not included in the analysis.

Finally, we performed a Pearson correlation analysis of $S$ with $\delta a, d$ and $\delta t$ using the statistical computing program " $R$ ".

\section{Results and discussion}

The Pearson correlations of $\delta a(\%), d$ (days) and $\delta t$ (days) with $S$ are shown in Figure 3. A $p<0.01$ level of significance was considered (Figure 3, blue line). Even though no correlation was found between $\delta a$ or $\delta t$ with $S$, stimulatingly, there was a significant positive correlation between $d$ and $S$, with a coefficient of 0.503 with a $p$ of approximately zero. In addition, we carried out linear regression, which fit the data reasonably and showed a reasonable coefficient of $R^{2} \sim 0.25$, as can be seen in Figure 4. Although both of these parameters are not high, they are statistically significant. Thus we consider this as an important result, as it shows a possible relationship between the radon anomaly duration, $d$, and both the magnitude of the impending earthquake and the distance from the earthquake epicenter to the measurement site. In other words, this reveals that for two seismic events with the same magnitude, the nearer one to the measurement site will show a longer anomaly than the farther one. This tends to confirm the physical hypotheses presented at the beginning, as it is expected that an event that occurs near to a measurement site should result in a stronger local

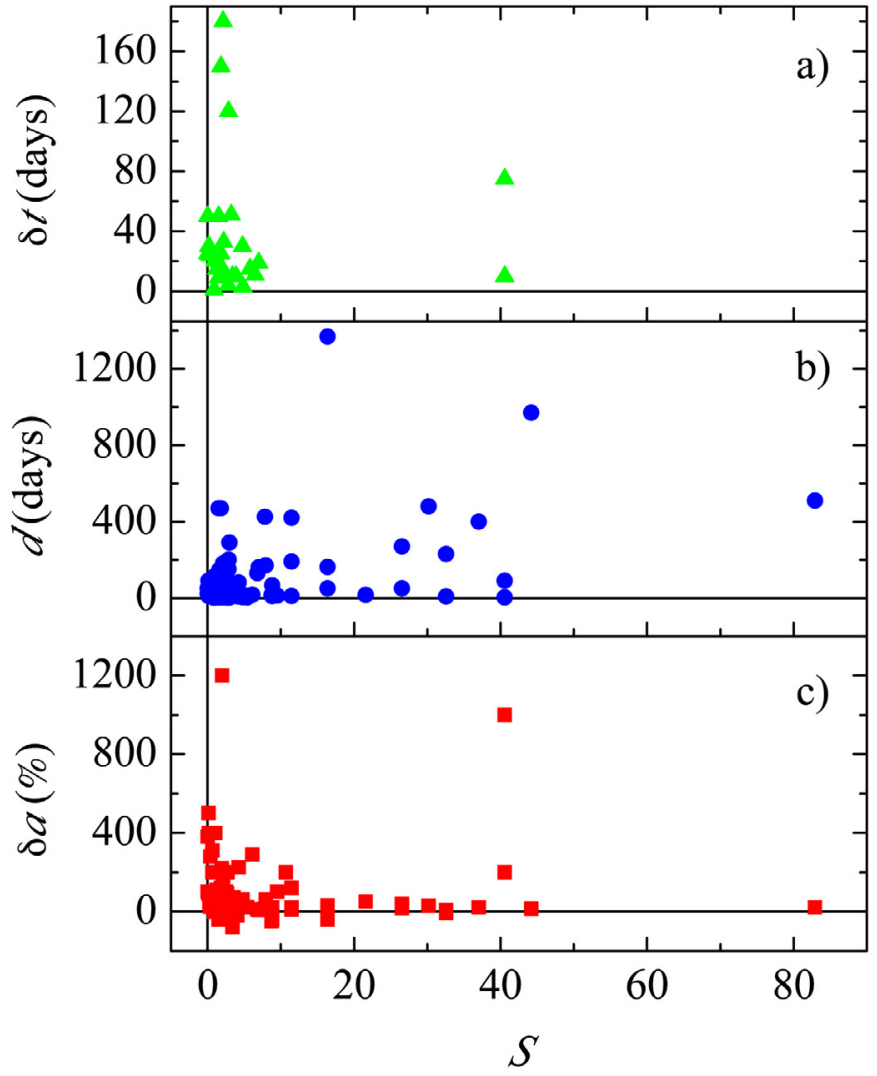

Figure 2. The three parameters of interest presented as functions of $S$ (defined in Equation 1). (a) The time interval between the beginning of the radon measurement anomalies and the occurrences of the earthquakes, $\delta t$ (days). (b) The duration of the anomalies, $d$ (days). (c) The relative amplitudes of the anomalies, $\delta a(\%)$. The data shown were extracted from Toutain and Baubron [1999].

deformation field, together with a larger increase in the soil permeability (while also implying a slower recovery to the equilibrium state), and this should induce a longer radon emanation episode, as compared with the farther event. Likewise, a stronger earthquake that has a larger preparation radius will tend to generate a radon source of larger superfi-

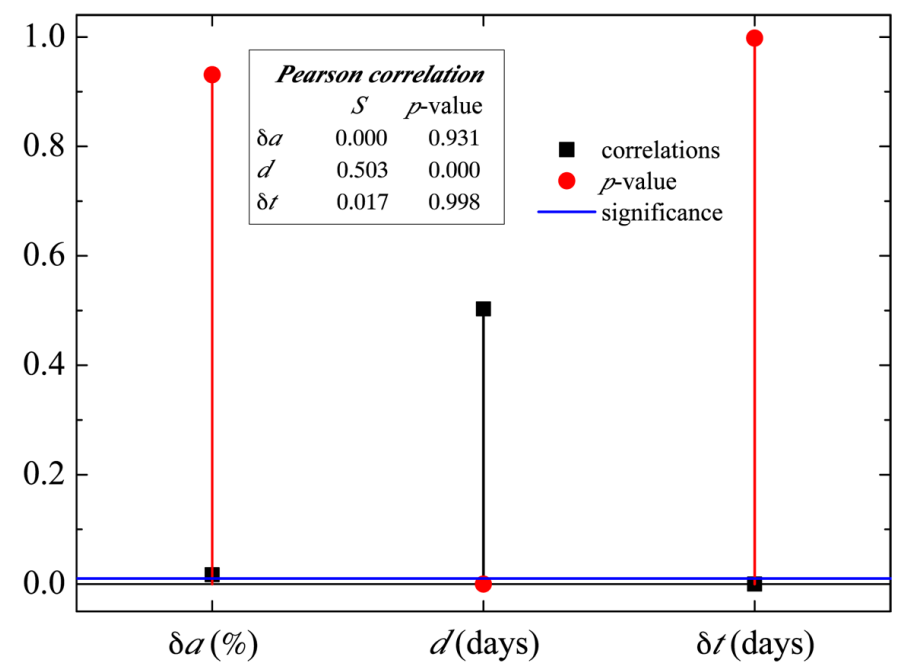

Figure 3. Results obtained for Pearson correlation tests for $\delta a, d$ and $\delta t$ with $S$ correlations. 


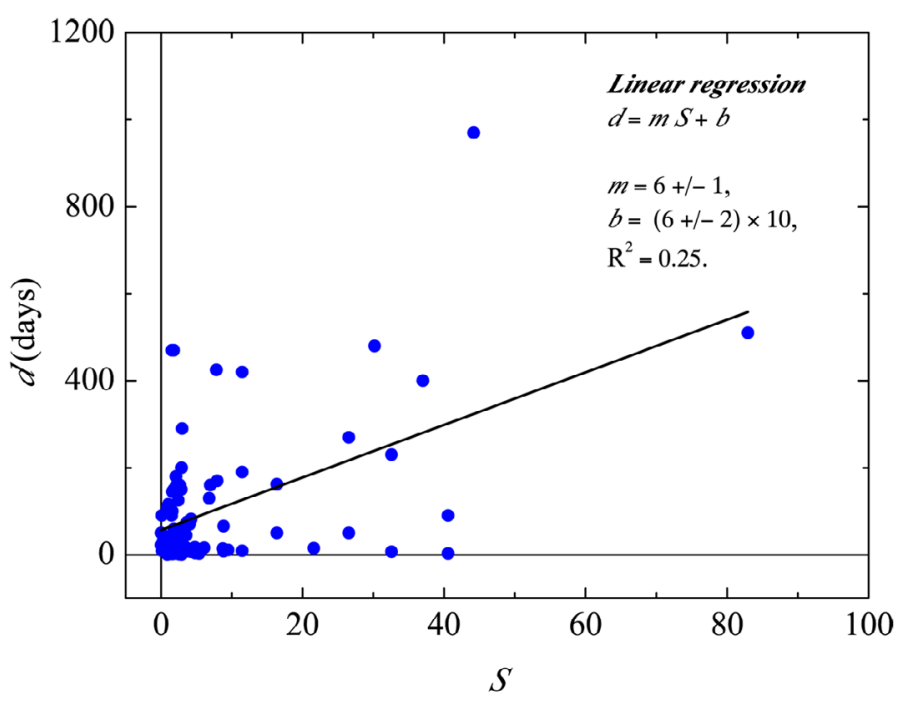

Figure 4. Linear regression of $d$ as a function of $S$.

cial area, together with anomalous radon levels (in both the soil and the groundwater) that last longer. It therefore appears that parameter $d$ is related more directly to the overall process of deformation and the permeability state than the other parameters. Indeed, the amplitude of the anomalies, $\delta a$, depends on the amount of radon released, which is unavoidably related to the specific lithology at the measuring site, such that comparisons of data from different sites has relatively little meaning. Likewise, the interval between the beginning of the anomaly and the occurrence of the earthquake, $\delta t$ (days), is strictly reliant on the intricate process of the radon source, the emanation and flow of the radon through the fissure network of the rocks, and its diffusion through the overlying soil at the observation site, such that, once again, the results from different sites are blurred by several uncontrolled site-related factors.

At this point, it is important to note that recorded radon anomalies are very much dependent on the local weather conditions; namely, the precipitation and water run-off, and also the temperature. In contrast, there is no relevant influence from the wind [Chen and Thomas 1994]. Actually, precipitation affects the soil permeability, therefore reducing the radon release rate, and this might be responsible for the short duration of radon anomalies like the points on the $S$ axis $(d \sim 0)$ in Figure $2 \mathrm{~b}$. Thus special care must be taken with meteorological conditions. Indeed, a possibility to minimize any meteorological influences on these radon anomalies is to consider only the anomalies with $S>S_{c}$ (a critical value to study only the events with the earthquake epicenter close enough to the measuring site; e.g., $S_{c}=20$ ). However, due to the restricted size of the database considered in the present study, this would significantly reduce the number of selected occurrences and thereby preclude the realization of any statistical analyses.

Unfortunately, as mentioned by Toutain and Baubron [1999], there is almost no information in the literature of very important parameters like the focal mechanics of these earthquakes, and the meteorological conditions during the anomalies, among others. Thus, the results shown here are preliminary and deeper investigations will be required to provide more robust results. In particular, the effect of the focal mechanism on the radon anomalies is definitely important, and although it requires a significant monitoring effort, it could complement the present analysis in the future. Nevertheless, stimulating perspectives for earthquake hazard assessment are found here, and they are presented at the end of this report (Section 4).

On the other hand, from a comparison between the results from an analysis performed by Cicerone et al. [2009] and the present study, it is interesting to note that a clear relationship between the radon anomalies and the earthquake parameters is found here, while no particular relationships were found by Cicerone et al. [2009]. From our point of view, this is because they did not take into account the balance between the distance of the earthquake epicenter to the measuring site and its magnitude; thus, it is not physically appropriate to compare anomalies from different magnitudes at different distances (see discussion in Section 2). For this reason, we plan to apply our methodology to the very relevant data that was compiled by Cicerone et al. [2009], and to compare their data with the present data. Indeed, new studies using larger databases with careful attention to meteorological conditions, and particularly using more advanced statically methods (like the Kolmogorov-Smirnov test), need to be carried out in the near future.

\section{Final remarks}

The present study constitutes a first step to a more profound analysis that must include a larger and more complete database of radon anomalies, with particular attention being paid to the meteorological conditions, and especially to precipitation (this is a crucial aspect for future studies). Nevertheless, by using a simple approach, this supports significant, although not decisive, evidence of a correlation between the seismic activity and the duration of the radon anomalies reported by Toutain and Baubron [1999]. However, the present viewpoint of many studies, e.g., Pulinets and Ouzounov [2011], tends to emphasize the analysis of satellite data, e.g., Sarkar et al. [2011], as a crucial approach to the study of earthquake precursory phenomena. We believe that similar to the case of meteorology, local stations are determining in the better constraining of models, and for that reason, much effort is still needed in multiple-parameter-based research of earthquake precursory phenomena at the local level. To this end, we suggest the combination of satellite InSAR analysis, which provides an accurate calculation of the deformation area (especially if compared to the rough estimation by the formula of Dobrovolsky et al. [1979]), with local radon measurements in seismically active regions. In this way, it will be possible for future studies to fully ex- 
plore the effects of deformation area on radon emissions, to provide more assertive results.

Acknowledgements. HGS acknowledges the support of two Portuguese institutions: the Science and Technology Foundation for the grant SFRH/BPD / 63880/2009, and the Calouste Gulbenkian Foundation for the grant Estímulo à Criatividade e à Qualidade na Actividade de Investigação in the science program of 2010. Important discussions with Prof R. Giles Harrison, Dr Cláudia Serrano, and Marta Melgão are strongly acknowledged. We are also thankful for the support of the Annals in Geophysics Editor, Prof Salvatore Barba, and the reviewers of our manuscript, Dr Valerio Curcio and Dr Anna Riggio; the final form of our manuscript was greatly enriched by their suggestions.

\section{References}

Biagi, P. F., L. Castellana, T. Maggipinto, R. Piccolo, A. Minafra, A. Ermini, S. Martellucci, C. Bellecci, G. Perna, V. Capozzi, O.A. Molchanov and M. Hayakawa (2006). LF radio anomalies revealed in Italy by the wavelet analysis: possible preseismic effects during 1997-1998, Phys. Chem. Earth, 31, 403-408.

Chauhan, V., O.P. Singh, V. Kushwah, V. Singh and B. Singh (2009). Ultra-low-frequency (ULF) and total electron content (TEC) anomalies observed at Agra and their association with regional earthquakes, J. Geodyn., 48, 68-74.

Chen, C., and D.M. Thomas (1994). Soil processes and chemical transport, J. Environ. Qual., 23, 173-179.

Cicerone, R.D., J. E. Ebel and J. Britton (2009). A systematic compilation of earthquake precursors, Tectonophysics, 476, 371-396.

Dobrovolsky, I.P., S.I. Zubkov and V.I. Miachkin, (1979). Estimation of the size of earthquake preparation zones, Pure Appl. Geophys., 117, 1025-1044.

Geller, R.J. (1991). Shake-up for earthquake prediction, Nature, 352, 275-276 (also 353, 612).

Harrison, R.G., K.L. Aplin and M.J. Rycroft (2010). Atmospheric electricity coupling between earthquake regions and the ionosphere, J. Atmos. Sol. Terr. Phys., 72, 376-381.

Hauksson, E. (1981). Radon content of groundwater as an earthquake precursor: evaluation of worldwide data and physical basis, J. Geophys. Res., 86, 9397-9410.

Kagan, Y.Y., and L. Knopoff, (1987). Statistical short-term earthquake prediction, Science, 236, 1563-1567.

Ouzounov, D., D. Liu, K. Chunli, G. Cervone, M. Kafatos and P. Taylor (2007). Outgoing long-wave radiation variability from IR satellite data prior to major earthquakes, Tectonophysics, 431, 211-220.

Pulinets, S., and D. Ouzounov (2011). Lithosphere-atmosphere-ionosphere coupling (LAIC) model - a unified concept for earthquake precursor validation, J. Asian Earth Sci., 41, 371-382.

Sarkar, S., S. Tiwari and A.K. Gwal (2011). Electron density anomalies associated with M 5.9 earthquakes in Indonesia during 2005 observed by DEMETER, J. Atmos. Solar Terr. Phys., 73, 2289-2299.
Silva, H.G., M. Bezzeghoud, A.H. Reis, R.N. Rosa, M. Tlemçani, A.A. Araújo, C. Serrano, J.F. Borges, B. Caldeira and P.F. Biagi (2011). Atmospheric electrical field decrease during the $M=4.1$ Sousel earthquake (Portugal), Nat. Hazards Earth Syst. Sci., 11, 987-991.

Silva, H.G., M.M. Oliveira, C. Serrano, M. Bezzeghoud, A.H. Reis, R. N. Rosa, P.F. Biagi (2012). Influence of seismic activity on the atmospheric electric field in Lisbon (Portugal) from 1955 to 1991, Annals of Geophysics, 55 (1), 193-197.

Toutain, J.P., and J.C. Baubron (1999). Gas geochemistry and seismotectonics: a review, Tectonophysics, 304, 1-27.

Virk, H.S., and S. Baljinder (1994). Radon recording of Uttarkashi earthquake, Geophys. Res. Lett. 21, 737-740.

Virk, H.S. (1995). Radon monitoring of microseismicity in the Kangra and Chamba Valleys of Himachal Pradesh, India, Nucl. Geophys., 9, 141-146.

Wyss, M., ed. (1991). Evaluation of Proposed Earthquake Precursors, 94 pp., AGU, Washington, D.C.; doi:10.1029/ SP032.

\footnotetext{
^ Corresponding author: Hugo Manuel Gonçalves da Silva, University of Évora, School of Sciences and Technology (ECT), Geophysical Centre of Évora and Physics Department, Évora, Portugal; email: hgsilva@uevora.pt.

(C) 2013 by the Istituto Nazionale di Geofisica e Vulcanologia. All rights reserved.
} 